\title{
Interacciones evolutivas como un posible mecanismo de interacción medicamentosa: una aproximación para el control de la resistencia bacteriana
}

\author{
Héctor Holguín, Pedro Amariles y William Ospina
}

\section{Evolutionary interactions as a possible mechanism of drug interactions: an approach to the control of antibiotic-resistant bacteria}

In the 21 st century, the world is facing a post-antibiotic era, in which the appearance of infections with a minor or no therapeutic alternative is common. In this context, it is essential to implement measures that optimize the available therapeutic tool, thus generating new strategies that strengthen the fight against growing bacterial resistance. The evolutionary interactions suggest that the development of sensitivity or resistance of a microorganism to an antimicrobial will have an inverse effect (collateral sensitivity) or similar (cross resistance) in a second antimicrobial that is involved in this system. The evolutionary interactions are considered as a new mechanism of drug interaction, and additional, it establishes the collateral sensitivity as a therapeutic strategy to combat bacterial resistance, which could be integrated and evaluated in the future as a new strategy in the antimicrobial stewardship programs.

Key words: Evolutionary interactions, collateral sensitivity, bacterial resistance, drug interactions, antimicrobial stewardship programs.

Palabras clave: Interacciones evolutivas, susceptibilidad colateral, resistencia microbiana, interacciones medicamentosas, programa de gerenciamiento de antimicrobianos.

\section{Introducción}

E 1 uso de los antimicrobianos remontado hacia los años 40', cambió radicalmente el tratamiento y curso de las enfermedades infecciosas. Alexander Fleming, tras descubrir la penicilina, aseguró que los microorganismos podrían volverse resistente a algunos antimicrobianos en relación con el proceso evolutivo natural de los seres vivos. De este modo, con su descubrimiento y uso, empiezan a identificarse microorganismos que, siendo susceptibles inicialmente, se tornan resistentes a determinados antimicrobianos ${ }^{1}$.

En el siglo XXI, la humanidad se encuentra frente a una era post-antibiótica, en la cual es común la aparición de infecciones con limitadas e incluso nulas alternativas terapéuticas. La resistencia ha alcanzado niveles alarmantes en varios países del mundo, generando la inefectividad de algunos antimicrobianos ${ }^{1}$. Los genes de resistencia portados por los microorganismos tienen la característica de propagarse rápidamente, convirtiendo infecciones que usualmente no eran complicadas, en verdaderos retos para la medicina moderna. La industria farmacéutica no ha invertido suficientes recursos de innovación y económicos, denotando bajo interés en el descubrimiento y desarrollo de moléculas con nuevos mecanismos de acción ${ }^{2,3}$. En este contexto, se hace indispensable implementar medidas que optimicen el arsenal terapéutico disponible hasta la fecha, con el fin de generar nuevas estrategias que fortalezcan la lucha contra la creciente resistencia bacteriana y la era post-antibiótica. Los programas de uso racional de antimicrobianos a través de los Programas de Gerenciamiento de Antimicrobianos (PGAn) se convierten en una alternativa para disminuir la resistencia a los antimicrobianos ${ }^{4}$.

Una interacción medicamentosa (IM) es una modificación cuantificable en la duración y magnitud del efecto de un medicamento, asociado a la administración previa o simultánea de otra sustancia: medicamento, alimentos, fitoterapéuticos, etc. ${ }^{5}$. En este marco, las interacciones evolutivas son poco aplicadas en la práctica clínica, dado su desconocimiento por parte del personal asistencial. Por tanto, el objetivo de esta revisión estructurada fue sistematizar la literatura científica disponible para establecer las interacciones evolutivas como un mecanismo de IM y explorar su uso como una estrategia para combatir la creciente resistencia bacteriana.

\section{Métodos}

Se realizó una búsqueda sistemática en Pubmed/ Medline, utilizando los siguientes términos MESH: (AntiInfective Agents OR Anti-Bacterial Agents OR Antifungal Agents OR Antiviral Agents) AND Collateral Sensitivity,
Universidad de Antioquia,

Colombia.

Grupo de investigación

Promoción y Prevención

Farmacéutica (HO, PA).

Departamento de Farmacia (PA).

Clínica Medellín S.A. (HO, WO)

El Grupo de Investigación

Promoción y Prevención

Farmacéutica recibió apoyo

económico de la convocatoria

de estrategia de sostenibilidad

2014-2015, del Comité para el

Desarrollo de la Investigación

(CODI) de la Universidad de

Antioquia, Medellín, Colombia.

Los autores manifiestan no tener conflicto potencial de interés.

Recibido: 7 de febrero de 2017 Aceptado: 24 de julio de 2017

Correspondencia a:

Héctor Holguín.

hector_holguin30@hotmail.com 
limitando la búsqueda a artículos en idioma inglés y español, artículos con acceso a texto completo y limitando la fecha hasta septiembre de 2016. Se excluyeron artículos que no abordaran el tema objetivo de la revisión sistemática. Los artículos incluidos fueron seleccionados por dos investigadores de forma independiente. Para ello, se revisaron los títulos y resúmenes de todos los artículos identificados para decidir su elegibilidad. Los artículos seleccionados se analizaron conjuntamente y, por consenso, se definió su inclusión o no.

\section{Resultados}

La búsqueda sistemática identificó 11 artículos de los cuales se excluyeron cuatro, debido a que carecían de relación con el objeto de la revisión sistemática. En total se incluyeron siete artículos a partir del criterio de búsqueda. Adicionalmente, a partir de las referencias de los artículos seleccionados, se identificaron 10 artículos de interés que se incluyeron en la revisión.

\section{Las interacciones evolutivas como causa de variabilidad en la respuesta terapéutica de los antimicrobianos}

Las IMs tradicionalmente se han clasificado en dos grandes grupos:

- Aquellas que tienen un mecanismo a nivel farmacocinético (las concentraciones plasmáticas del medicamento se ven afectadas por variaciones a nivel de la absorción, distribución, metabolismo y excreción del medicamento) y

- Las IMs que se presentan a nivel farmacodinámico (se presenta modificación en la respuesta terapéutica, sin modificaciones en las concentraciones plasmáticas), también conocidas como interacciones fisiológicas ${ }^{6}$.

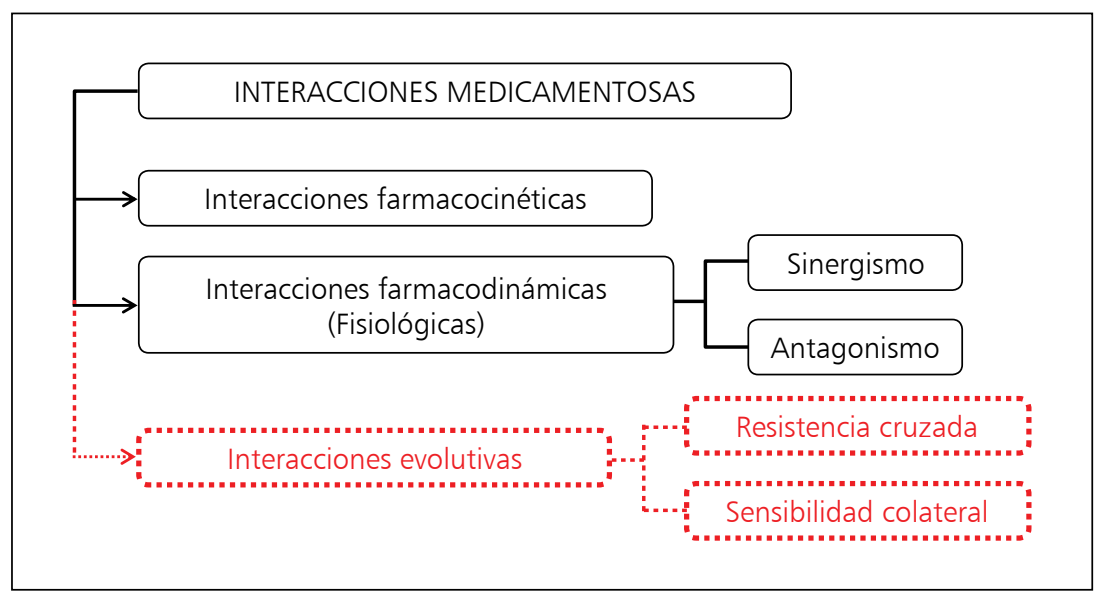

Figura 1. Mecanismo de las interacciones medicamentosas de los antimicrobianos.
A su vez, las IMs farmacodinámicas se subdividen en IMs sinérgicas, donde el efecto terapéutico producido, generado por la combinación de dos medicamentos, es mayor a la suma de los efectos de cada uno de los medicamentos, lo que se traduce en un mayor efecto terapéutico $\mathrm{y}$, por ende, siendo ésta una experiencia exitosa en el tratamiento del virus de la inmunodeficiencia humana (VIH) y tuberculosis ${ }^{7}$; y las IMs antagónicas, que se presentan con la disminución del efecto terapéutico debido a la combinación de dos medicamentos, en comparación con la suma del efecto de cada medicamento por separado.

Relacionado con los antimicrobianos, la modificación en el efecto terapéutico de un antimicrobiano puede aparecer como consecuencia a la exposición previa a un antimicrobiano, concepto excluido de las definiciones de las IMs farmacocinéticas y farmacodinámicas ${ }^{6}$.

Las interacciones evolutivas establecen que el desarrollo de susceptibilidad o resistencia de un microorganismo a un antimicrobiano tendrán un efecto inverso o similar en un segundo antimicrobiano que se incorpore a este sistema. Por ello, se puede observar que la exposición de un microorganismo a un antimicrobiano A con posterior desarrollo de resistencia al mismo, se traduzca inmediatamente en el desarrollo de resistencia a un antimicrobiano $\mathrm{B}$, sin exposición previa del antimicrobiano B al microorganismo, lo que se conoce como resistencia cruzada. Por el contrario, se puede observar que el desarrollo de resistencia del antimicrobiano A por parte de un microorganismo, aumenta la susceptibilidad al microorganismo a un antimicrobiano $\mathrm{B}$, término conocido como susceptibilidad colateral. Los cambios en las interacciones evolutivas se presentan por modificaciones en los genes que confieren resistencia a los antimicrobianos $^{8}$.

En este contexto, en la Figura 1 se esquematiza la propuesta de los mecanismos de IM para los antimicrobianos, la cual incluye las interacciones evolutivas como proceso con capacidad de modificar la respuesta terapéutica de los antimicrobianos.

En la susceptibilidad colateral, las modificaciones en los perfiles de susceptibilidad pueden ocurrir de forma bidireccional (el aumento de la resistencia al antimicrobiano A se representa en aumento de la susceptibilidad al antimicrobiano B y viceversa) o unidireccional (el aumento de la resistencia al antimicrobiano A se representa en aumento de la susceptibilidad al antimicrobiano $\mathrm{B}$, pero no de forma recíproca).

Características particulares de las interacciones evolutivas:

- La resistencia cruzada se presenta entre sustancias químicas pertenecientes al mismo grupo químico y al menos a un medicamento de una clase diferente, mientras que la susceptibilidad cruzada se presenta entre sustancias químicamente diferentes ${ }^{8}$. 
- Las interacciones evolutivas disminuyen la adquisición de mecanismos de resistencia comparado con el uso de cada una de las moléculas por separado ${ }^{9} \mathrm{y}$

- Se ha demostrado que las combinaciones de medicamentos que presentaban susceptibilidad colateral conllevaban menos resistencia comparada con combinaciones de medicamentos que no presentaban susceptibilidad colateral ${ }^{7}$.

El campo de la susceptibilidad colateral ha sido ampliamente aplicada en las áreas de oncología desde los años $70^{10}$, donde se ha demostrado que en células de ovario de hámster chino que expresan bombas de expulsión como la glicoproteína-P (confiriendo resistencia a algunos quimioterapéuticos), medicamentos con efecto inhibitorio sobre la glicoproteína-P (por ejemplo, verapamilo), aumentan la sensibilidad de los medicamentos a los cuales se era previamente resistente, llegando incluyendo a niveles se susceptibilidad superiores a los basales para la misma línea celular estudiada ${ }^{11}$.

\section{Efecto de los mecanismos de resistencia en la homeostasia microbiana}

La adquisición de mecanismos de resistencia representa en muchos casos, costos físicos para la bacteria, como lo demostró un estudio que evidenció la disminución hasta de $41 \%$ en la tasa de crecimiento in vitro de Escherichia coli cuando adquirió algún mecanismo de resistencia, en comparación con las cepas salvajes ${ }^{12}$.

Gagneux y cols. ${ }^{13}$, demostraron que las cepas de Mycobacterium tuberculosis que contenían el gen rpob, el que confiere resistencia a rifampicina, implicó la alteración en la homeostasia de la bacteria en comparación con sus símiles sensibles. Dada esta característica de inestabilidad física para $M$. tuberculosis, a través de mecanismos de compensación, para la adquisición de una nueva característica (por ejemplo, un mecanismo de resistencia adicional), este microorganismo deberá perder de igual forma otra característica previamente adquirida. De esta forma, la teoría de la compensación explica la susceptibilidad colateral ${ }^{14}$.

\section{La susceptibilidad colateral como estrategia frente a la resistencia a antimicrobianos}

Se han planteado tres mecanismos por los cuales un microorganismo puede desarrollar susceptibilidad colateral:

- Las mutaciones o alteraciones de la membrana en los cuales se puede disminuir el transporte activo de uno de ellos, y por otro lado, se puede presentar una disminución en la actividad de las bombas de expulsión para otros antimicrobianos. Los aminoglucósidos realizan el transporte de membrana de una manera activa a través del Proton-Motive Force (PMF), sistema de la cual dependen bombas de expulsión de muchos antimicrobianos. En este sentido, mutaciones que eviten el ingreso de aminoglucósidos por el sistema de transporte activo PMF, disminuirán de igual forma la actividad de las bombas de expulsión lo cual permite que los antimicrobianos dependientes de estas bombas tengan una mayor concentración intra-bacteriana ${ }^{15}$.

- La alteración de las actividades reguladas por el ADN, llevan a que la bacteria cambie su fenotipo (pleiotropismo) y, por ende, se presente susceptibilidad a otros antimicrobianos. Este mecanismo es presentado principalmente por aquellos antimicrobianos cuya actividad farmacológica se centra en el ADN (quinolonas).

- Dos antimicrobianos que son afines a la misma enzima pueden presentar susceptibilidad colateral.

La susceptibilidad colateral se caracteriza por presentar disminuciones en la CIM hasta de ocho veces con respecto a la cepa salvaje, caso contrario con lo que ocurre en la resistencia cruzada. Por ejemplo, las quinolonas y los $\beta$-lactámicos son antimicrobianos que han demostrado presentar resistencia cruzada, siendo el incremento de la CIM hasta de 32 veces el valor basal (de la cepa salvaje). En este sentido, la elección incorrecta de los antimicrobianos puede generar un fallo terapéutico a corto plazo.

Para el estudio in vitro de la susceptibilidad colateral, se expone un microorganismo con un perfil de susceptibilidad conocido, a un medio de cultivo con una concentración de un antimicrobiano. Una vez el microorganismo desarrolle resistencia al antimicrobiano que se encuentra expuesto, se evalúan los perfiles de susceptibilidad del microorganismo a los antimicrobianos de interés y nuevamente se inocula el microorganismo en un nuevo medio de cultivo con el mismo antimicrobiano del medio de cultivo previo, pero en una concentración mayor, y así sucesivamente como se representa en la Figura 2.

Lazar y cols. ${ }^{14}$, evaluaron en E. coli, la susceptibilidad cruzada de 24 antimicrobianos distribuidos de la siguiente manera: 4 con actividad frente a la pared bacteriana, 3 con acción frente a la ADN girasa, 5 aminoglucósidos, 5 inhibidores de la síntesis de proteínas, 2 con actividad frente a la síntesis de ácido fólico y 3 antimicrobianos con actividad frente a grampositivos. Se inició la evaluación con concentraciones antimicrobianas sub-inhibitorias, las cuales se incrementaron cada 4 días por un período de 240384 generaciones. Las poblaciones alcanzaron concentraciones de resistencia de 20-328 veces de incremento de la CIM. Con las cepas resistentes se midieron los cambios en las susceptibilidades a los demás antimicrobianos no expuestos. Se encontró que $35 \%$ de los pares de antimicrobianos investigados mostraron susceptibilidad cruzada al menos en una dirección (la susceptibilidad colateral no ocurrió entre pares de antimicrobianos con el mismo blanco molecular), 44\% de los pares de antimicrobianos evaluados involucró a aminoglucósidos, quienes aumen- 


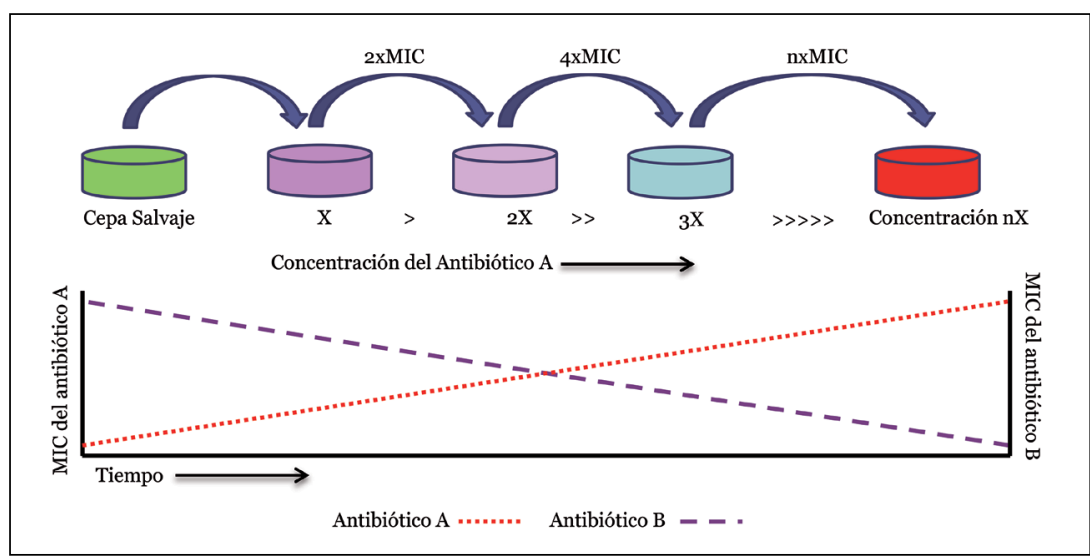

Figura 2. Ilustración de la sensibilidad colateral. Esta figura muestra cómo se desarrollan los estudios de sensibilidad colateral, donde se toma una cepa bacteriana sin ningún mecanismo de resistencia y se somete a concentraciones crecientes de un antimicrobiano. Con posterioridad a la exposición al antimicrobiano, se toma una muestra de la cepa y se evalúa la Concentración Inhibitoria Mínima para los antimicrobianos de interés $y$, al graficarlas, se puede observar el comportamiento del microorganismo en las diferentes concentraciones.

taron la susceptibilidad de antimicrobianos inhibidores de la síntesis de ADN, síntesis de pared e inhibidores de síntesis de proteínas y se observaron disminuciones de dos a 10 veces la CIM con respecto a las cepas de referencia.

Gonzales y cols. ${ }^{16}$, evaluaron combinaciones de tres antimicrobianos (piperacilina, meropenem y el inhibidor de $\beta$-lactamasa, tazobactam) en varios rangos de concentraciones de estos tres medicamentos, encontrando una fuerte susceptibilidad colateral entre meropenem y piperacilina/tazobactam y entre piperacilina y meropenem/ tazobactam. Se estableció que piperacilina/tazobactam sensibiliza a meropenem frente a Staphylococcus aureus resistente a meticilina-SARM, pero no de manera recíproca. La susceptibilidad colateral a piperacilina también fue conferida previa exposición a tazobactam, pero no viceversa. En este estudio no se encontró susceptibilidad colateral a tazobactam después de la exposición a cualquiera sustancia simple o combinada. En un sub-análisis, piperacilina mostró susceptibilidad colateral a imipenem. Ninguna de las cefalosporinas probadas mostró susceptibilidad colateral para el carbapenem/penicilina/inhibidor de $\beta$-lactamasa evaluado.

Se probaron in vitro cuatro cepas de Acinetobacter baumannii multi-resistente, que contenían unos de los genes que alteran la biosíntesis de la membrana externa de lipopolisacáridos $(\operatorname{lpxA}$, lpxC y lpxD) con el objetivo de evaluar la susceptibilidad con antimicrobianos clínicamente relevantes (por ejemplo, colistín). Una vez establecida la resistencia a colistín, se observaron aumentos en la susceptibilidad de amikacina (4-16 veces), ceftazidima (4-64 veces), imipenem (4-32 veces), cefepime (0-8 veces) y meropenem (4-64 veces) dada la pérdida del li- popolisacárido. Los cambios más significativos se vieron, con respecto al valor basal, en azitromicina ( $>32$ veces), rifampicina ( $>16$ veces) y vancomicina ( $64-512$ veces $)^{17}$.

Tugce y cols. ${ }^{18}$, ensayaron 88 cepas de $E$. coli durante 21 días frente a 22 antimicrobianos clínicamente relevantes, encontrando susceptibilidad cruzada, principalmente para aquellas combinaciones de antimicrobianos que incluían un aminoglucósido. Se encontró que $80 \%$ de las cepas que presentaron susceptibilidad cruzada incluyendo un aminoglucósido, contenían una mutación en el gen TrkH que codificó una proteína transportadora de iones potasio, sugiriendo así que este gen es el responsable de conferir resistencia a aminoglucósidos y conferir susceptibilidad a otros antimicrobianos. Se propuso que la mutación en el gen TrkH antagonizó la actividad de la bomba de expulsión PMF, con lo cual se lograron concentraciones terapéuticas a nivel citoplasmático de antimicrobianos que son expulsados por este sistema.

Xiaoliang y cols. ${ }^{19}$, demostraron en $S$. aureus que la PBP2c codificada por el gen recientemente descrito mecC, no media resistencia a penicilina y que el gen blaZ tipo E es requerido para la resistencia a penicilina. De tal forma, se puede lograr efecto bactericida de penicilina (o amoxicilina) en combinación con ácido clavulanico para superar el efecto de las penicilinasas producidas a través de blaZ, siendo el efecto similar al esperado con vancomina. $S$. aureus RM portando el gen $\operatorname{mec} C$ ha sido comúnmente encontrado en el ganado, muy posiblemente asociado a la presión selectiva determinada por el uso rutinario de cefalosporinas de primera y tercera generación para el tratamiento y prevención de mastitis.

\section{La susceptibilidad colateral y la rotación de antimicrobianos como una nueva estrategia terapéutica frente a la resistencia antimicrobiana}

Los principios del sistema de rotación de antimicrobianos se basan en que, siendo la resistencia bacteriana favorecida por la exposición a un antimicrobiano, con la abstinencia o ausencia de uso de esa molécula puntual y acorde a la teoría de la compensación, hay una pérdida del mecanismo de resistencia frente a lo cual, el microorganismo podrá nuevamente presentar un fenotipo de susceptibilidad donde este fármaco inicial puede presentar nuevamente beneficios terapéuticos, como se evidencia en la Figura 3.

La rotación de estos fármacos puede incluir el número de antimicrobianos que sean considerados, siendo una característica de este sistema, que el medicamento inicial debe ser administrado nuevamente al final del ciclo, contemplando así el inicio de un nuevo ciclo ${ }^{8}$.

Con respecto a la rotación de antimicrobianos, Imamovic y cols. ${ }^{8}$, desarrollaron un estudio donde concluyeron que aquellas bacterias que presentaban resistencia a aminoglucósidos, presentaron incremento en la sus- 
ceptibilidad para 14 antimicrobianos químicamente no relacionados, pertenecientes principalmente al grupo de los $\beta$-lactámicos, polimixinas, macrólidos, tetraciclinas, anfenicoles, fosfomicina y nitrofurantoina. La susceptibilidad colateral no tiene un esquema definido, lo cual se evidenció para las cepas resistentes a amikacina, quienes mostraron aumento de la CIM hasta en dos veces para ampicilina, amoxicilina y hasta de 16 veces para cefuroxima; mientras que las cepas resistentes a gentamicina y kanamicina no mostraron aumentos de la susceptibilidad para ampicilina y amoxicilina y sólo presentaron aumento de cuatro veces para cefuroxima. Adicionalmente, las cepas resistentes a ampicilina no presentaron aumento de la CIM para amikacina, gentamicina o kanamicina como sí es el caso inverso, lo cual demuestra que la susceptibilidad cruzada no es un proceso bidireccional en todos los casos.

Las combinaciones de antimicrobianos de amikacina presentaron bajos cambios evolucionarios con respecto a las combinaciones sin amikacina $(\mathrm{p}<0,05)$. Por ello se concluyó que la combinación de medicamentos es una estrategia que disminuye la evolución de la resistencia bacteriana ${ }^{7}$.

Kim y cols. ${ }^{20}$, evaluaron 120 cepas de $S$. aureus frente a trimetoprim, neomicina y ciprofloxacina evaluando modelos de predicción de susceptibilidad colateral. Para ello, sometieron las cepas de $S$. aureus a los tres antimicrobianos por 22 días con el siguiente esquema: antimicrobianos únicos (sólo un antimicrobiano frente a una cepa), mezclados (dos antimicrobianos simultáneos) o alternados (alternación temporal de dos antimicrobianos). Se realizaron rotaciones de antimicrobianos cada $20 \mathrm{~h}$ buscando altos niveles de resistencia. Pasado este tiempo, las bacterias nuevamente se sometieron a concentraciones más altas hasta alcanzar 10 réplicas del tratamiento. Este estudio demostró que la rotación de antimicrobianos disminuyó la tasa total de evolución de resistencia comparada con el tratamiento individual. La rotación entre trimetoprim y neomicina disminuyó la tasa de adquisición de resistencia a trimetoprim en $41 \%$ (p: 0,0003). Alternando neomicina y ciprofloxacina, se disminuyó la resistencia a ciprofloxacina en 54\% (p: 0,0003) y alternando trimetoprim y ciprofloxacina disminuyó la resistencia a ciprofloxacina en $40 \%$ (p: 0,003). Se observaron menos mutaciones en grlA en el tratamiento con ciprofloxacinaneomicina ( $\mathrm{p}$ : 0,0061), menos mutaciones en folA con la combinación trimetoprim-neomicina (50\%, p: 0,01687). Finalmente, los mutantes resistentes a trimetoprim mostraron susceptibilidad colateral a ciprofloxacina pero no viceversa. También se ha demostrado susceptibilidad colateral entre eritromicina y ácido fusídico para S. aureus en modelos in vitro ${ }^{21}$.

Aunque, los resultados de utilización de la estrategia de rotación de antimicrobianos son limitados, un estudio retrospectivo fue realizado con el objetivo de evaluar

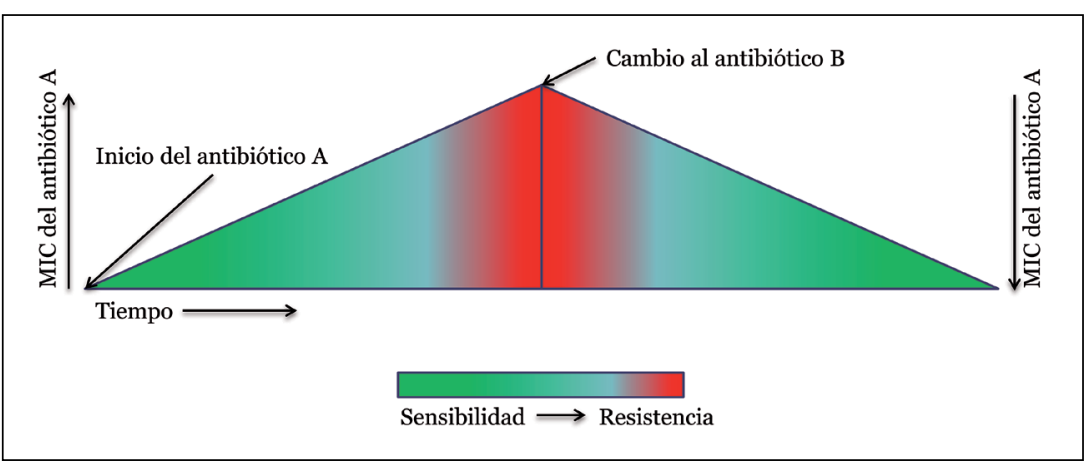

Figura 3. Esquema que representa un programa de rotación de antimicrobianos. Cuando el microorganismo, inicialmente sensible, adquiere resistencia al antimicrobiano $A$, se hace la rotación al antimicrobiano B, lo cual lleva a una disminución de la presión por parte del antimicrobiano A sobre el microorganismo y por ende, hay una pérdida en los genes de resistencia del antimicrobiano A, por el alto costo físico que implica mantener este mecanismo mientras es presionado por el antimicrobiano $B$.

los resultados de la implementación de un modelo de rotación de antimicrobianos, por nueve años, en una unidad de cuidados intensivos quirúrgica (UCI-Q), utilizando como control una unidad de cuidados intensivos médicos (UCI-M) (ambas unidades con 16 camas). En dicho estudio, en la UCI-Q se implementó la rotación mensual de piperacilina/tazobactam, imipenem/cilastatina y ceftazidima; mientras que dicho protocolo no fue implementado en la UCI-M. Con posterioridad al análisis retrospectivo, se encontró que la rotación de antimicrobianos sobre Pseudomonas aeruginosa aumentó la susceptibilidad a ceftazidima (de 66 a 81\%, p: 0,003) y piperacilina/tazobactam (de 75 a 85\%, p: 0,021), mientras que la susceptibilidad a imipenem/cilastatina permaneció inalterada (p: 0,989). En la UCI-M utilizada como control, los perfiles de susceptibilidad de $P$. aeruginosa permanecieron estables, excepto para imipenem/cilastatina, el que disminuyó significativamente (de 71 a $52 \%$, p: 0,01). De igual forma, para $E$. coli en la UCI-Q, la susceptibilidad a piperacilina/tazobactam presentó aumento (de 46 a $83 \%$, p: 0,025 ), mientras que no se presentaron modificaciones significativas en los perfiles de susceptibilidad para ceftazidima (p: 0,23) ni imipenem/cilastatina (100\% susceptibilidad). Para Klebsiella pneumoniae no hubo diferencias estadísticamente significativas en la UCI-Q para los antimicrobianos evaluados. La media mensual de la prescripción de estos antimicrobianos duplicó el valor para la prescripción en la UCI-M comparada con la UCI$\mathrm{Q}$ con respecto a piperacilina/tazobactam $(\mathrm{p}<0,0001)$ e imipenem/cilastatina $(p>0,0001)$. Caso contrario, ceftazidima no tuvo diferencias estadísticamente significativas entre las dos unidades ${ }^{22}$.

Las primeras aproximaciones realizadas para correlacionar los hallazgos in vitro con los microorganismos 
aislados en el ambiente hospitalario, han demostrado que la susceptibilidad colateral encontrada en el laboratorio no corresponde completamente a los hallazgos detectados en los microorganismos clínicos. Adicionalmente, se encontró que aquellos antimicrobianos que expresaban susceptibilidad colateral tenían una tasa de depuración mayor de la infección ( 1,5 veces) cuando se trataban infecciones con microorganismos resistentes a gentamicina y tratadas con cefuroxima y viceversa, que en infecciones con microorganismos resistentes a amikacina y tratadas con ciprofloxacina ${ }^{23}$.

\section{Discusión}

Las interacciones evolutivas podrían ser consideradas como un nuevo mecanismo de IM, debido a que en su desarrollo existe una modificación en el perfil de susceptibilidad de un antimicrobiano, (variación en la magnitud del efecto de un medicamento), asociado a la administración previa de otro antimicrobiano. Este nuevo mecanismo de IM propuesto no es explicado en los mecanismos farmacocinéticos y farmacodinámicos, tradicionalmente planteados ${ }^{5}$.

La susceptibilidad colateral se contempla como una estrategia terapéutica para combatir la resistencia bacteriana, que se podría integrar y evaluar en un futuro como una nueva estrategia en los PGAn ${ }^{8}$.

Los aminoglucósidos son los antimicrobianos que tienen más soporte en la literatura científica sobre su uso en susceptibilidad colateral. Por ejemplo, los aminoglucósidos son una estrategia terapéutica para el manejo de bacterias gramnegativas portadoras de $\beta$-lactamasa de espectro extendido y SARM. Bajo las bases teóricas de la susceptibilidad colateral, el uso de aminoglucósidos contra estos microorganismos podría mejorar la susceptibilidad a otros antimicrobianos que se presentarían como una nueva alternativa terapéutica y así, se retrasaría la aparición de bacterias multi-resistentes.

Las aplicaciones clínicas de la susceptibilidad colateral en antimicrobianos presenta algunas limitantes al día de hoy: los estudios realizados hasta el momento son llevados a cabo in vitro, los cuales tienen la limitante de obviar el hospedero de la infección y, por ende, todo el proceso farmacocinético y farmacodinámico del medicamento en el organismo, el sistema inmune del hospedero y la actividad de éste frente a la bacteria, por lo cual hay dificultades en la extrapolación de estos estudios a seres humanos; adicional, el uso de dos antimicrobianos para combatir una infección puede conllevar el aumento de reacciones adversas medicamentosas en el paciente y un aumento significativo de los costos asociados en salud, variables que podrían incluso superar los potenciales beneficios de susceptibilidad colateral.

La aproximación clínica a la rotación de antimicrobianos y la susceptibilidad colateral como una estrategia para la disminución de la resistencia antimicrobiana, requiere que se consideren las discrepancias de los resultados presentados con microorganismos de referencia y los microorganismos clínicamente aislados. Estas diferencias pueden ser atribuidas a que, la resistencia por microorganismos aislados en el ambiente clínico esté asociada a plásmidos con varios mecanismos de resistencia; mientras que in vitro, la adquisición de la resistencia puede ser más controlada (dada la exposición a un único antimicrobiano, por ejemplo). En este contexto, se deben realizar estudios clínicos que permitan hacer evaluar el impacto de la susceptibilidad colateral en la resistencia bacteriana.

\section{Resumen}

En el siglo XXI, la humanidad se encuentra frente a una era post-antibiótica, en la cual es común la aparición de infecciones con una menor o nula alternativa terapéutica. En este contexto, se hace indispensable implementar medidas que optimicen el arsenal terapéutico disponible, generando nuevas estrategias para contrarrestar la creciente resistencia bacteriana y la era post-antibiótica. Las interacciones evolutivas plantean que el desarrollo de susceptibilidad o resistencia de un microorganismo a un antimicrobiano tendrá un efecto inverso (susceptibilidad colateral) o similar (resistencia cruzada) en un segundo antimicrobiano incorporado en este sistema. Las interacciones evolutivas se plantean como un nuevo mecanismo de interacción medicamentosa, al tiempo que se identifica a la susceptibilidad colateral como una estrategia terapéutica para combatir la resistencia bacteriana, que se podría integrar y evaluar en un futuro como una nueva estrategia en los programas de gerenciamiento de antimicrobianos. 


\section{Referencias bibliográficas}

1.- World Health Organization, editor. Antimicrobial resistance: global report on surveillance. Geneva, Switzerland: World Health Organization; 2014. 232 p.

2.- Freire-Moran L, Aronsson B, Manz C, Gyssens I C, So A D, Monnet D L, et al; ECDC-EMA Working Group. Critical shortage of new antibiotics in development against multidrug-resistant bacteria-Time to react is now. Drug Resist Updat 2011; 14 (2): 118-24.

3.- Premanandh J, Samara B S, Mazen A N. Race against antimicrobial resistance requires coordinated action - An overview. Front Microbiol 2016; 6: 1536.

4.- Collins C D, Miller D E, Kenney R M, Mynatt R P, Tiberg M D, Cole K, et al. The state of antimicrobial stewardship in Michigan: Results of a statewide survey on antimicrobial stewardship efforts in acute care hospitals. Hosp Pharm 2015; 50 (3): 180-4.

5.- Interacciones medicamentosas. En: Amariles P, editor. El medicamento. Compendio básico para su utilización correcta. Medellín: Impresos Ltda; 2002. p. 155-246.

6.- Baym M, Stone LK, Kishony R. Multidrug evolutionary strategies to reverse antibiotic resistance. Science 2016; 351 (6268): aad3292.

7.- Munck C, Gumpert H K, Wallin A I, Wang $\mathrm{H} \mathrm{H}$, Sommer M O. Prediction of resistance development against drug combinations by collateral responses to component drugs. Sci Transl Med 2014; 6 (262): 262ra156.

8.- Imamovic L, Sommer M O. Use of collateral sensitivity networks to design drug cycling protocols that avoid resistance development. Sci Transl Med 2013; 5 (204): 204ra132.
9.- $\quad$ Suzuki S, Horinouchi T, Furusawa C. Suppression of antibiotic resistance acquisition by combined use of antibiotics. J Biosci Bioeng 2015; 120 (4): 467-9.

10.- Hall M D, Handley M D, Gottesman M M. Is resistance useless? Multidrug resistance and collateral sensitivity. Trends Pharmacol Sci 2009; 30 (10): 546-56

11.- Tsuruo T, Iida H, Tsukagoshi S, Sakurai Y. Increased accumulation of vincristine and adriamycin in drug-resistant p388 tumor cells following incubation with calcium antagonists and calmodulin inhibitors. Cancer Res 1982; 42: 4730-3.

12.- Lázár V, Nagy I, Spohn R, Csörgő B, Györkei Á, Nyerges Á, et al. Genome-wide analysis captures the determinants of the antibiotic cross-resistance interaction network. Nat Commun 2014; 5: 4352.

13.- Gagneux S, Long C D, Small P M, Van T, Schoolnik G K, Bohannan B J. The competitive cost of antibiotic resistance in Mycobacterium tuberculosis. Science 2006; 312 (5782): 1944-6.

14.- Pál C, Papp B, Lázár V. Collateral sensitivity of antibiotic-resistant microbes. Trends Microbiol 2015; 23 (7): 401-7.

15.- Lázár V, Pal Singh G, Spohn R, Nagy I, Horváth B, Hrtyan M, et al. Bacterial evolution of antibiotic hypersensitivity. Mol Syst Biol 2013; 9: 700.

16.- Gonzales P R, Pesesky M W, Bouley R, Ballard A, Biddy B A, Suckow M A, et al. Synergistic, collaterally sensitive $\beta$-lactam combinations suppress resistance in MRSA. Nat Chem Biol 2015; 11 (11): 855-61.

17.- García-Quintanilla $M$, Carretero-Ledesma $M$, Moreno-Martínez P, Martín-Peña R, Pachón J, McConnell M J. Lipopolysaccharide loss produces partial colistin dependence and collateral sensitivity to azithromycin, rifampicin and vancomycin in Acinetobacter baumannii. Int J Antimicrob Agents 2015; 46 (6): 696-702.

18.- Oz T, Guvenek A, Yildiz S, Karaboga E, Tamer Y T, Mumcuyan N, et al. Strength of selection pressure is an important parameter contributing to the complexity of antibiotic resistance evolution. Mol Biol Evol 2014; 31 (9): 2387-401.

19.- Ba X, Harrison E M, Lovering A L, Gleadall N, Zadoks R, Parkhill J, et al. Old drugs to treat resistant bugs: methicillin resistant Staphylococcus aureus isolates with mecC are susceptible to a combination of penicillin and clavulanic acid. Antimicrob Agents Chemother 2015; 59 (12): 7396-404.

20.- Kim S, Lieberman T D, Kishony R. Alternating antibiotic treatments constrain evolutionary paths to multidrug resistance. Proc Natl Acad Sci USA 2014; 111 (40): 14494-9.

21.- Rodríguez de Evgrafov M, Gumpert H, Munck C, Thomsen T T, Sommer M O. Collateral resistance and sensitivity modulate evolution of high-level resistance to drug combination treatment in Staphylococcus aureus. Mol Biol Evol 2015; 32 (5): 1175-85.

22.- Sarraf-Yazdi S, Sharpe M, Bennett K M, Dotson T L, Anderson D J, Vaslef S N. Impact of antibiotic cycling on susceptibility profiles of gram-negative pathogens in an intensive care unit: a nine-year retrospective review. J Surg Res 2012; 176 (2): e73-8.

23.- Obolski U, Dellus-Gur E, Stein GY, Hadany L. Antibiotic cross-resistance in the lab and resistance co-occurrence in the clinic: Discrepancies and implications in E. coli. Infect Genet Evol 2016; 40: 155-61. 